\title{
Insufficient Fruit and Vegetable Intake in a Low- and Middle-Income Setting: A Population-Based Survey in Semi-Urban Tanzania
}

\author{
Beverly Msambichaka 1,2,3 , Ikenna C. Eze 2,3 (1), Ramadhani Abdul ${ }^{1}$, Salim Abdulla ${ }^{1}$, \\ Paul Klatser ${ }^{4}$, Marcel Tanner ${ }^{2,3}$, Ramaiya Kaushik ${ }^{5}$, Eveline Geubbels ${ }^{1}$ and \\ Nicole Probst-Hensch 2,3,* \\ 1 Ifakara Health Institute Dar es Salaam, Kiko Avenue, P.O. Box 78373, Dar es Salaam, Tanzania; \\ msambichakabeverly@gmail.com (B.M.); rabdul@ihi.or.tz (R.A.); sabdulla@ihi.or.tz (S.A.); \\ egeubbels@ihi.or.tz (E.G.) \\ 2 Swiss Tropical and Public Health Institute, Socinstrasse 57, 4051 Basel, Switzerland; \\ ikenna.eze@swisstph.ch (I.C.E.); marcel.tanner@swisstph.ch (M.T.) \\ 3 University of Basel, Petersplatz 1, 4001 Basel, Switzerland \\ 4 Athena Institute, Vrije Universiteit/Free University, 1081 HV Amsterdam, The Netherlands; \\ paulklatser@gmail.com \\ 5 Shree Hindu Mandal Hospital, Chusi St, P.O. Box 581, Dar es Salaam, Tanzania; ceo@hindumandal.org \\ * Correspondence: nicole.probst@swisstph.ch; Tel.: +41-612-848-378
}

Received: 30 December 2017; Accepted: 13 February 2018; Published: 16 February 2018

\begin{abstract}
A daily intake of 5 portions of fruit and vegetables (FV) is recommended for protection against non-communicable diseases (NCDs). Inadequate FV intake is a global problem but resource-poor countries like Tanzania are most deprived and constitute settings where little is known for informing public health interventions. This study aimed to describe the prevalence of inadequate FV intake, frequency of FV intake, portions of FV intake and their associations with socio-demographic/lifestyle factors in South-Eastern Tanzania. Data on FV dietary indicators, socio-demographic factors, smoking, alcohol and healthcare use were collected from 7953 participants ( $\geq 15$ years) of the population-based MZIMA open community cohort (2012-2013). Multivariable logistic regression was used to examine associations between FV intake outcomes and their socio-demographic/lifestyle determinants. Most (82\%) of the participants did not meet the recommended daily FV intake While only a fraction consumed fruits daily $(15.5 \%)$, almost half consumed vegetables daily $(44.2 \%)$. However, the median (IQR) number of vegetable portions consumed was lower (2(1)/person/day) than that for fruits (2(2)/person/day) People with higher education were more likely to consume fruits daily. Independent correlates of inadequate FV intake included young age, being male, low education, low-income occupations, low alcohol, high tobacco and low healthcare use. Public health interventions should target the socio-economically deprived and culturally-rooted preferences while prioritizing promotion of vegetable for most immediate gain in overall FV intake.
\end{abstract}

Keywords: fruit and vegetables; education; occupation; healthcare use; Ifakara; Tanzania

\section{Introduction}

A daily intake of fruits and vegetables (FV) is recommended for protection against almost all major non-communicable diseases (NCDs) [1-4]. FV have vitamins, minerals and fiber which either singly or synergistically protects against NCDs as well as communicable diseases $[4,5]$. Antioxidants found in FV can prevent the action of carcinogens by inhibiting oxidative DNA damage [6]. Furthermore, vitamins B12, B6 as well as B9 help reduce levels of homocysteine and risk of cardiovascular diseases 
(CVDs) [7,8]. Many FV are also rich in potassium which helps modulate blood pressure $[9,10]$. Maximum benefits from FV could be attained by daily consumption of five portions (400 g) [11,12].

Despite promising benefits from FV consumption, more than $75 \%$ of the global population do not consume sufficient FV [13] and according to the most recent estimate, this accounted for $2.9 \%$ of all lives lost in 2009 [14]. A STEPwise approach to surveillance (STEPS) survey conducted in 2012 showed that almost all Tanzanians ( $>95 \%$ ) consumed insufficient FV [15]. Similar findings were found in neighboring Malawi [16], Zambia [17], Mozambique [18] and Botswana [19]. The STEPS survey findings provide useful information on the scale of the problem but do not allow better characterization of FV intake and susceptibility factors to inadequate FV consumption. Depending on the context, inadequate FV intake may be attributed more to lower fruit intake [20,21], or lower vegetable intake [22]. Hence, it was recently suggested that studies on FV consumption should consider fruits separately from vegetables given that separate interventions may be needed $[18,23,24]$. According to the literature, most African countries do not have food-based dietary guidelines and the few countries with food based dietary guidelines are not explicit on how much FV should be consumed [25-29]. This is noteworthy because people can only act in favor of good health if they are aware, are convinced and know how to act [30,31]. In Tanzania, the Ministry of Health and Social Welfare promotes two important elements of FV intake, first is daily consumption and second is consumption in high quantities. Shortage of detailed evidence on FV consumption practices hampers targeted responses at policy and health system levels to promote FV consumption [32].

Socio-demographic determinants are important in shaping FV intake patterns [33,34]. Education and wealth, as indicators of socio-economic status (SES) are related to FV intake [13,35]. Education exposes people to necessary health information [36] and builds capacity for comprehension of existing recommendations [31,37-39], both of which could be limiting factors when absent [23]. People with more education can secure higher paying jobs which may help address issues of "affordability," a critical barrier in most developing countries $[18,21,40]$. Variations in the social, cultural and structural environment of different occupations may also pose different constraints to FV consumption. Lifestyle factors like alcohol consumption and smoking may also in principle affect FV intake where an increased intake may be a compensatory behavior to heavy drinking or smoking [41]. Although this has been explored by Western studies with other lifestyle factors [42,43], evidence from an African population is limited. Although the use of health services may also influence lifestyle choices and vice versa partly through preventive counseling [44], there is limited evidence on how healthcare use influences FV intake.

Evidence on the importance of socio-demographic determinants in FV consumption may help guide public health responses that are population-specific. In this study, we aimed to describe the patterns of FV consumption and explored how these patterns associate with socio-demographic and lifestyle factors in southeastern Tanzania, using data from the MZIMA open community cohort.

\section{Materials and Methods}

\subsection{Study Design and Participants}

The MZIMA open community cohort is lodged within the Ifakara Urban Health and Demographic Surveillance System (IU-HDSS) [45]. The IU-HDSS is a longitudinal database that collects information on demographic and vital events including births, deaths and migration. The MZIMA cohort was created in 2012 to study among others, changes in NCD burden and their determinants over time [46]. Information collected in the cohort includes socio-demographic characteristics, NCD risk factors such as FV consumption, smoking, alcohol habits as well as health care use.

Community sensitization activities were conducted and these included meetings with community leaders, pamphlets, radio spots, sensitization at community events. Door-to-door visits, following prior notification by the ten-cell leader, the lowest level of local administration, were conducted. Eligible participants were $\geq 15$ years, willing and able to give informed consent and resident in the study areas 
during the latest IU-HDSS round. In the first study round, a total of 8734 participants were recruited from June 2012 to April 2013 from two areas of the IU-HDSS (Mlabani and Viwanja Sitini). Community members in the study area are a mix of indigenous inhabitants and migrants. Both Mlabani and Viwanja Sitini are parts of Ifakara ward, situated in Morogoro Region, southeast Tanzania.

\subsection{Data Collection}

Interviews were conducted at participants' homes between June 2012 and August 2013 by trained interviewees using a structured questionnaire. The interview tool was translated from English into Swahili and back translated to English and was piloted. Interviewers used tablet personal computers programmed with the open-source Open Data Kit [47]. Automated validation and skip patterns were programmed to minimize faulty data entries. Interviewers also kept field diaries for problems that occurred during data collection. These sheets were reviewed by the supervisor, who made suggestions for improvement, at the end of each day.

Classification of FV consumption.

Participants were asked questions on their intake of fruits and intake of vegetables using questions from the WHO STEPS survey tool for NCD risk factors [48]. Questions covered frequency of consumption in a typical week and number of standard portions on days of consumption. A standard portion equals $80 \mathrm{~g}$. Medium size fruits like an orange, an apple, a banana, a pear counted for one portion. Other fruits like half an avocado, half a large mango also formed one standard portion. A typically very large watermelon accounted for 16 portions. One small glass (150 mls) of $100 \%$ fruit juice was equal to one portion of fruit. Three heaped tablespoons ( $30 \mathrm{~g}$ per heaped spoon) of cooked vegetables were equal to one portion. Sometimes people used small bowls for relish, which was equivalent to two standard portions if it were cooked vegetables, or one standard portion if it were fresh salad. Interviewers used picture cards with common fruits and vegetables found in the study setting. The picture cards were used to help participants recall on FV intake in the past week but also to help them minimize errors in estimation of standard portions consumed. In order to get the average daily portions of fruits and portions of vegetables, we multiplied the number of days of consumption and the number of portions consumed in a typical day and divided by seven. Participants who reportedly consumed fruits or vegetables every day in a typical week were classified as having "daily fruit intake" or "daily vegetables intake," respectively. People who consumed fruits or vegetables on a less than daily basis were categorized as "no daily fruit intake" or "no daily vegetable intake," respectively. Those without fruit or vegetable intake in a usual week were classified as "no fruit intake" or "no vegetable intake," respectively. Participants who ate less than 5 portions of fruits and/or vegetables per day were categorized as having inadequate FV intake.

\subsection{Covariate Information}

Participants were interviewed for their age (years), sex (male/female), marital status (single/ monogamous/polygamous/widowed/separated), migration status (migrant/non-migrant), regions of ethnic affiliation (Morogoro/Iringa/Shinyanga/Kilimanjaro/Ruvuma/Coast/Mbeya/Others) and religion (Muslim/Catholic/Lutheran/Others) as previously described [46]. Participants were also asked about their educational attainment and occupation. Educational level was categorized as: no formal education; primary education (up to 7 years of formal education); secondary education (7-13 years of formal education); and tertiary education ( $>13$ years of formal education) [46]. Phrasing for social determinants listed above was adapted from standard questions used in Analyzing Longitudinal Population-based HIV / AIDS data for Africa (ALPHA) network [49].

Occupational status was categorized as follows: "unemployed" being those who have no income generating activity; farming, fishing and livestock keeping; owning a small business (employing $<5$ persons); owning a large business (employing $\geq 5$ persons); professionals (white collar jobs); skilled manual labors (including drivers, carpenters, etc.); and unskilled manual labors (including menial jobs). Participants were also asked if they smoked or consumed alcohol in the past 12 months [48]. Smoking 
status was categorized into never, former and current smokers while alcohol use was categorized into daily and not daily, for exploratory purposes, based on the available data. Information on frequency of healthcare visits (hospital, dispensary or home-based care worker) in the past 12 months was also collected and categorized into none, one, two, three, four, five and six or more visits, which allowed for investigating dose-response relationship with FV intake. Participants were also asked if they have been diagnosed with diabetes or hypertension, or any cardiovascular disease in order to derive NCD variable, assigning yes to the presence of any of the three diseases and no to the absence of all three.

\subsection{Statistical Analyses}

We described the study population stratified by sex. We tabulated the FV intake according to socio demographic characteristics and healthcare use. Using three outcome variables- "less than daily fruit intake versus daily fruit intake," "less than daily vegetable intake versus daily vegetable intake" and "inadequate FV intake versus adequate FV intake," we applied logistic regression to explore the independent association of these outcome variables with sex, age group, marital status, educational level, occupation, ethnicity, religion and migration status, using mutually-adjusted models. In a further step using the adjusted socio-demographic model, we explored associations of FV intake with lifestyle characteristics and healthcare use habits. Data analyses excluded participants with missing data as well as those with doctor-diagnosed hypertension, diabetes or cardiovascular diseases (Figure 1). The intention for NCD exclusion was to capture trends of FV intake in apparently healthy individuals, towards prevention. All statistical analyses were done using STATA Version 14 (STATA Corporation, Texas). Associations between these outcome variables and socio-demographic and lifestyle determinants are presented as odds ratios (OR) and their $95 \%$ confidence intervals. Associations were considered significant at $p$ value $<0.05$.

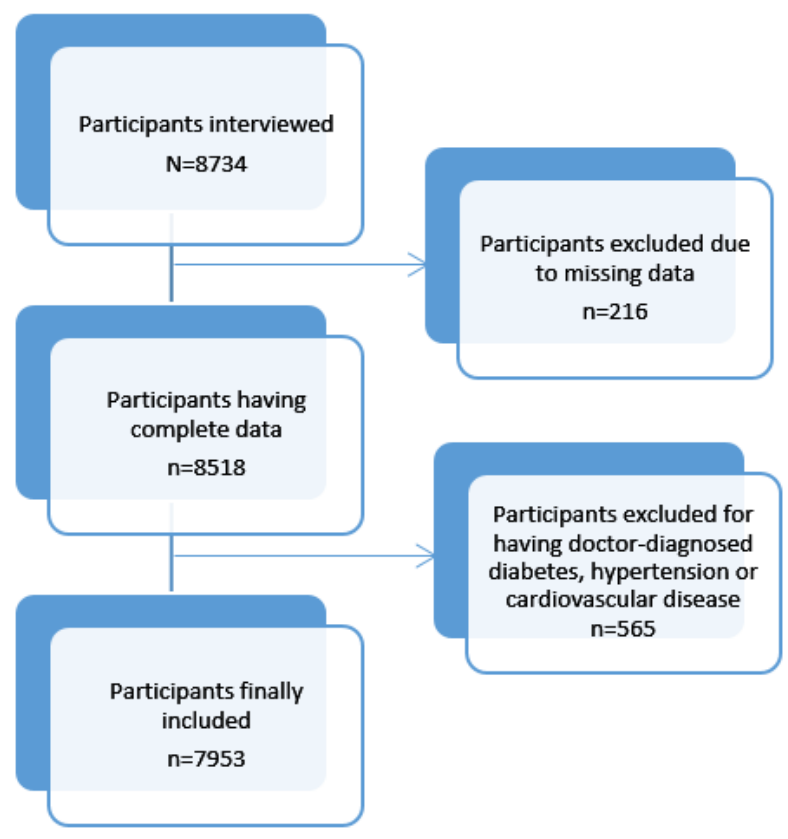

Figure 1. Participant selection flow chart.

\subsection{Ethics Consideration}

The MZIMA open community cohort was approved by the Ifakara Health Institute Institutional Review Board and the National Institute for Medical Research with reference numbers IHI/IRB/AM/01-2014 and NIMR/HQ/R.8a1Vol. IX/I320 respectively. All participants provided informed written consent to participate in the study. Confidentiality of participants' identity was ensured by use of encrypted identification codes and proper storage of personal information. 


\section{Results}

\subsection{Population Description}

Out of 8734 ( $\geq 15$ years) enrolled, 8518 had complete information and 565 participants with confirmed NCDs were excluded bringing the total number of participants for the present analyses to 7953 (Figure 1). Women comprised 64.3\% of the study population. Participants below 18 years comprised $27 \%$ of those below 25 years and $10 \%$ of the entire study population. More than half (55.2\%) of the participants were educated at primary level and more women than men had not received any formal education. More than half of all participants were engaged in an income-generating activity $(59.8 \%)$ and were mainly farmers (25.4\%). Major ethnic groups included Morogoro, Iringa and Ruvuma. Alcohol consumption and smoking rates were generally low. (Table 1).

Table 1. Description of the study population.

\begin{tabular}{|c|c|c|c|c|c|}
\hline Variables & Groups & All \% $(N)$ & Males \% $(N)$ & Females \% $(N)$ & Chi- Squared Test \\
\hline \multirow{3}{*}{ Age } & Below 25 years & $39.1(3111)$ & $36.2(1027)$ & $40.8(2084)$ & \multirow{3}{*}{$<0.001$} \\
\hline & $50-60$ years & $6.7(535)$ & $7.3(206)$ & $6.4(329)$ & \\
\hline & 60 and above & $9.0(719)$ & $11.1(314)$ & $7.9(405)$ & \\
\hline \multirow{2}{*}{ Education } & No Formal Education & $14.1(1118)$ & $9.1(258)$ & $16.8(860)$ & \multirow{2}{*}{$<0.001$} \\
\hline & Tertiary Education & $3.3(262)$ & $5.0(141)$ & $2.4(121)$ & \\
\hline \multirow{4}{*}{ Marriage } & Never married & $38.8(3088)$ & $46.6(1324)$ & $34.5(1764)$ & \multirow{4}{*}{$<0.001$} \\
\hline & Monogamous & $47.4(3770)$ & $44.7(1268)$ & $48.9(2502)$ & \\
\hline & Polygamous & $1.2(94)$ & $1.1(30)$ & $1.3(64)$ & \\
\hline & Widowed & $5.8(461)$ & $2.4(68)$ & $7.7(393)$ & \\
\hline \multirow{7}{*}{ Occupation } & Farming, Fishing, Livestock keeping & $25.4(2017)$ & $26.9(763)$ & $24.5(1254)$ & \multirow{7}{*}{$<0.001$} \\
\hline & Small business & $15.4(1221)$ & $14.8(421)$ & $15.6(800)$ & \\
\hline & Large business & $1.2(95)$ & $2.3(66)$ & $0.6(29)$ & \\
\hline & Professionals & $4.7(367)$ & $6.5(183)$ & $3.6(184)$ & \\
\hline & Skilled manual labor & $7.9(625)$ & $12.4(352)$ & $5.3(273)$ & \\
\hline & Unskilled manual labor & $5.4(429)$ & $9.2(262)$ & $3.3(167)$ & \\
\hline & Not working & $40.2(3199)$ & $27.9(792)$ & $47.1(2407)$ & \\
\hline \multirow{4}{*}{ Religion } & Muslim & $37.1(2952)$ & $38.2(1085)$ & $36.5(1867)$ & \multirow{4}{*}{0.258} \\
\hline & Catholic & $54.3(4317)$ & $53.6(1521)$ & $54.7(2796)$ & \\
\hline & Lutheran & $1.9(147)$ & $1.6(44)$ & $2.0(103)$ & \\
\hline & Other beliefs & $6.8(537)$ & $6.7(189)$ & $6.8(348)$ & \\
\hline \multirow{3}{*}{ Ethnicity } & Ruvuma region & $14.8(1179)$ & $14.2(403)$ & $15.2(776)$ & \multirow{3}{*}{$<0.001$} \\
\hline & Other regions & $12.8(1020)$ & $14.7(418)$ & $11.8(602)$ & \\
\hline & Morogoro region & $43.0(3422)$ & 40.5 (1147) & $44.5(2273)$ & \\
\hline
\end{tabular}


Table 1. Cont.

\begin{tabular}{|c|c|c|c|c|c|}
\hline Variables & Groups & All \% $(N)$ & Males \% $(N)$ & Females \% $(N)$ & Chi- Squared Test \\
\hline Alcohol & Daily & $2.4(189)$ & $3.3(93)$ & $1.9(96)$ & $<0.001$ \\
\hline \multirow[t]{2}{*}{ Smoking } & Former smoker & $3.1(245)$ & $6.5(183)$ & $1.2(62)$ & \multirow[t]{2}{*}{$<0.001$} \\
\hline & Current smoker & $7.3(577)$ & $17.2(487)$ & $1.8(90)$ & \\
\hline \multirow{4}{*}{ Healthcare use } & Two visits & $12.6(1004)$ & $11.6(328)$ & $13.2(676)$ & \multirow{4}{*}{$<0.001$} \\
\hline & Three visits & $11.8(935)$ & $10.7(305)$ & $12.3(630)$ & \\
\hline & Four visits & $7.4(590)$ & $5.5(157)$ & $8.5(433)$ & \\
\hline & Five visits & $2.7(214)$ & $2.3(64)$ & $2.9(150)$ & \\
\hline
\end{tabular}

N/A: not applicable. The chi-squared test compares proportions between males and females.

\subsection{Patterns of FV Consumption}

Inadequate FV consumption was observed in $82 \%$ of the study population (Table 2), i.e., the prevalence of not meeting the recommendation for daily eating of fruits or vegetables. Fruits tended to be consumed less frequently than vegetables. However, median vegetable portions were smaller than median portions of fruits (Table 2). Inadequate FV consumption appeared less prevalent as people became more educated, as well as in people who have higher-earning occupations. Inadequate FV consumption also tended to decrease with increasing use of healthcare services (Table 2).

Almost the entire study population consumed at least some vegetables $(98.5 \%)$ in the seven days preceding the interview. Vegetable consumption revealed two main patterns; "Daily vegetable intake" $(44.2 \%)$ and "No daily vegetable intake" (54.3\%). Median (IQR) vegetable portions consumed per day per person was 2 (1) portions. Older participants tended to have higher number of standard vegetable portions than younger people. The frequency of daily vegetable intake appeared to be lowest among least educated, unskilled and skilled laborers. Participants with more frequent use of healthcare also tended to have higher proportion of daily vegetable consumption when compared to those with less frequent health care use (Table 2).

The median (IQR) fruit portions consumed per day per person was 2 (2) portions. For fruits, three patterns emerged "Daily fruit intake" (15.5\%), "No daily fruit intake" (71.7\%) and "No fruit intake" $(12.7 \%)$. Younger participants appeared to consume more fruits daily $(16.1 \%)$ compared to older participants $(8.1 \%)$. The oldest age group had the lowest median (IQR) fruit portions (1 (1) portions). Figure 2 shows that participants tended to consume more FV with higher educational level, irrespective of gender. 
Table 2. Frequency and Patterns of Fruit and Vegetable Intake in the MZIMA Cohort, $N=7953$.

\begin{tabular}{|c|c|c|c|c|c|c|c|c|c|c|c|}
\hline Variable & Groups & $\begin{array}{l}\text { Daily Fruit } \\
\text { Intake a \% } \\
(N)\end{array}$ & $\begin{array}{l}\text { No Daily Fruit } \\
\text { Intake }{ }^{\mathrm{b}} \%(N)\end{array}$ & $\begin{array}{l}\text { No Fruit } \\
\text { Intake }{ }^{c} \% \\
(N)\end{array}$ & $\begin{array}{c}\text { Daily Fruit } \\
\text { Portions (Median } \\
\text { (IQR)) }\end{array}$ & $\begin{array}{c}\text { Daily } \\
\text { Vegetable } \\
\text { Intake }{ }^{\mathrm{a} \%}(N)\end{array}$ & $\begin{array}{c}\text { No Daily } \\
\text { Vegetable } \\
\text { Intake } \mathbf{b} \%(N)\end{array}$ & $\begin{array}{l}\text { No Vegetable } \\
\text { Intake }^{\mathrm{c}} \%(N)\end{array}$ & $\begin{array}{c}\text { Daily Vegetable } \\
\text { Portions (Median } \\
\text { (IQR)) }\end{array}$ & $\begin{array}{c}\text { Inadequate } \\
\text { FV Intake }{ }^{d} \\
(\%)\end{array}$ & $\begin{array}{l}\text { Chi } \\
\text { Squared } \\
\text { Test }\end{array}$ \\
\hline All & N/A & $15.4(1227)$ & $72.1(5734)$ & $12.5(992)$ & $2(2)$ & $44.2(3516)$ & $54.3(4318)$ & 1.5 (119) & $2(1)$ & 82 & $\mathrm{~N} / \mathrm{A}$ \\
\hline \multirow{2}{*}{ Sex } & Male & $15.6(440)$ & 72.7 (2064) & 11.8 (335) & $2(2)$ & 34.7 (985) & $62.8(1782)$ & $2.5(72)$ & $1(1)$ & 83 & \multirow{2}{*}{0.269} \\
\hline & Female & $15.4(787)$ & $71.8(3670)$ & $12.9(657)$ & $2(2)$ & 49.5 & $49.6(2536)$ & $0.9(47)$ & $2(1)$ & 82 & \\
\hline \multirow{4}{*}{ Age } & Below 25 & $16.1(502)$ & 72.5 (2254) & $11.4(355)$ & $2(2)$ & 36.1 (1124) & 62.1 (1932) & $1.8(55)$ & $1(1)$ & 84 & \multirow{4}{*}{$<0.001$} \\
\hline & $25-50$ & $15.9(572)$ & $72.6(2603)$ & 11.5 (413) & $2(2)$ & 46.4 (1665) & 52.1 (1872) & $1.4(51)$ & $2(1)$ & 82 & \\
\hline & $50-60$ & 17.8 (95) & $65.6(351)$ & $16.6(89)$ & $2(2)$ & $55.2(397)$ & 43.7 (314) & $0.9(5)$ & $2(1)$ & 76 & \\
\hline & Above 60 & $8.1(58)$ & $73.2(526)$ & 18.8 (135) & $1(1)$ & $55.2(397)$ & 43.7 (314) & 1.1 (8) & $2(1)$ & 84 & \\
\hline \multirow{4}{*}{ Education } & No Education & $7.3(81)$ & 71.1 (795) & $21.7(242)$ & $1(1)$ & $51.3(573)$ & 47.1 (527) & $1.6(18)$ & $2(1)$ & 86 & \multirow{4}{*}{0.002} \\
\hline & Primary & $14.4(631)$ & 73.7 (3231) & $12.0(525)$ & $2(2)$ & 45.5 (1996) & $53.3(2336)$ & $1.3(55)$ & $2(1)$ & 82 & \\
\hline & Secondary & $20.2(441)$ & 70.3 (1537) & $9.5(208)$ & $2(2)$ & 38.1 (883) & $60.0(1312)$ & $1.9(41)$ & $1(1)$ & 82 & \\
\hline & Tertiary & $28.2(74)$ & $65.3(171)$ & $6.5(17)$ & $2(2)$ & 43.5 (114) & $54.6(143)$ & $1.9(5)$ & 1 (1) & 77 & \\
\hline \multirow{5}{*}{$\begin{array}{c}\text { Marital } \\
\text { status }\end{array}$} & Never married & 15.7 (485) & 72.6 (2243) & $11.7(360)$ & $2(2)$ & 36.2 (1117) & 61.7 (1904) & $2.2(67)$ & $1(1)$ & 84 & \multirow{5}{*}{0.001} \\
\hline & Monogamous & $16.3(627)$ & 72.1 (2718) & $11.3(425)$ & $2(2)$ & 47.6 (1796) & 51.4 (1937) & $1.0(37)$ & $2(1)$ & 81 & \\
\hline & Polygamous & $14.9(14)$ & $69.2(65)$ & $16.0(15)$ & $2(2)$ & $55.3(52)$ & 43.6 (41) & $1.1(1)$ & $2(1)$ & 79 & \\
\hline & Widowed & $8.0(37)$ & $69.2(319)$ & 22.8 (105) & $1(1)$ & 59.4 (274) & $39.3(181)$ & $1.3(6)$ & $2(1)$ & 84 & \\
\hline & Divorced & $11.9(64)$ & $72.0(389)$ & $16.1(87)$ & $1(2)$ & $51.3(277)$ & $47.2(255)$ & $1.5(8)$ & $2(1)$ & 85 & \\
\hline \multirow{2}{*}{$\begin{array}{l}\text { Work } \\
\text { status }\end{array}$} & Working & $16.4(780)$ & 71.8 (3415) & $11.8(559)$ & $2(2)$ & 47.4 (2252) & $51.3(2437)$ & $1.4(65)$ & $2(1)$ & 80 & \multirow[b]{2}{*}{$<0.001$} \\
\hline & Not working & $14.0(447)$ & $72.5(2319)$ & $13.5(433)$ & $2(2)$ & 39.5 (1264) & $58.8(1881)$ & $1.7(54)$ & $2(1)$ & 85 & \\
\hline \multirow{7}{*}{ Occupation } & Farming, Fishing, Livestock & $12.0(241)$ & $75.8(1528)$ & $12.3(248)$ & $2(2)$ & $53.1(1070)$ & $46.0(928)$ & $0.9(19)$ & $2(1)$ & 82 & \multirow{7}{*}{$<0.001$} \\
\hline & Small business & $18.0(221)$ & $71.7(876)$ & $10.2(124)$ & $2(2)$ & $45.5(556)$ & $53.2(649)$ & $1.3(16)$ & $2(1)$ & 79 & \\
\hline & Large business & $30.5(29)$ & $62.1(59)$ & $7.4(7)$ & $2(3)$ & $47.4(45)$ & $49.5(47)$ & $3.2(3)$ & $1(1)$ & 71 & \\
\hline & Professionals & $28.6(105)$ & $64.0(235)$ & $7.4(27)$ & $2(3)$ & $47.7(175)$ & $52.0(191)$ & $0.3(1)$ & $1(1)$ & 73 & \\
\hline & Skilled manual labor & $20.4(129)$ & $66.9(418)$ & $12.5(78)$ & $2(2)$ & $40.0(250)$ & $58.7(367)$ & $1.3(8)$ & $2(1)$ & 80 & \\
\hline & Unskilled manual labor & $12.8(55)$ & $66.7(299)$ & $17.5(7)$ & $2(2)$ & $36.4(156)$ & $59.4(255)$ & $4.2(18)$ & $2(1)$ & 87 & \\
\hline & Not working & $14.0(447)$ & $72.5(2319)$ & 13.5 (433) & $2(2)$ & 39.5 (1264) & $58.8(1881)$ & $1.7(54)$ & $2(1)$ & 85 & \\
\hline \multirow{4}{*}{ Religion } & Muslim & $15.6(459)$ & $71.8(2119)$ & $12.7(374)$ & $2(2)$ & 44.7 (1319) & $53.6(1582)$ & $1.7(51)$ & $2(1)$ & 82 & \multirow{4}{*}{0.934} \\
\hline & Catholic & $14.9(641)$ & $72.8(3141)$ & $12.4(535)$ & $2(2)$ & $44.5(1922)$ & $54.3(2345)$ & $1.2(50)$ & $1(1)$ & 83 & \\
\hline & Lutheran & $24.5(36)$ & $60.5(89)$ & $15.0(22)$ & $2(2)$ & $49.0(72)$ & $50.3(74)$ & $0.7(1)$ & $1(1)$ & 84 & \\
\hline & Others & $17.0(91)$ & $71.7(385)$ & $11.4(61)$ & $2(2)$ & $37.8(203)$ & $59.0(317)$ & $3.2(17)$ & $1(1)$ & 83 & \\
\hline \multirow{2}{*}{ Migration } & Non-migrants & $16.0(527)$ & $71.4(2357)$ & $12.6(417)$ & $2(2)$ & $44.8(1478)$ & $54.2(1788)$ & $1.1(35)$ & $2(1)$ & 82 & \multirow[b]{2}{*}{0.151} \\
\hline & Migrants & $15.0(700)$ & $72.6(3377)$ & $12.4(575)$ & $2(2)$ & 43.8 (2038) & $54.4(2530)$ & $1.8(84)$ & $2(1)$ & 83 & \\
\hline \multirow{8}{*}{ Ethnicity } & Morogoro region & $14.2(485)$ & $72.2(2471)$ & $13.6(466)$ & $2(2)$ & $48.6(1664)$ & $50.4(1723)$ & $1(35)$ & $2(1)$ & 82 & \multirow{8}{*}{0.140} \\
\hline & Iringa region & $14.6(132)$ & $73.8(667)$ & $11.6(105)$ & $2(2)$ & $41.0(371)$ & $58.2(526)$ & $0.7(7)$ & $1(1)$ & 83 & \\
\hline & Shinyanga/Mwanza/Tabora & $13.9(76)$ & $76.2(415)$ & $9.9(54)$ & $2(2)$ & $30.8(168)$ & $65.5(357)$ & $3.7(20)$ & $2(1)$ & 85 & \\
\hline & Kilimanjaro/Arusha region & $24.2(64)$ & $67.6(179)$ & $8.3(22)$ & $2(3)$ & $37.4(99)$ & $60.4(160)$ & $2.3(6)$ & $2(1)$ & 78 & \\
\hline & Ruvuma region & $15.4(181)$ & $72.2(851)$ & $12.5(147)$ & $2(2)$ & $45.3(534)$ & $53.7(633)$ & $1(12)$ & $1(1)$ & 81 & \\
\hline & Coast region & $14.0(71)$ & $70.9(348)$ & $14.7(72)$ & $2(2)$ & $45.4(223)$ & $52.6(258)$ & $2.0(10)$ & $2(1)$ & 84 & \\
\hline & Mbeya region & $17.3(22)$ & $74.8(95)$ & $7.9(10)$ & $2(2)$ & $32.3(41)$ & $66.1(84)$ & $1.6(2)$ & $2(1)$ & 87 & \\
\hline & Other regions & $19.2(196)$ & $69.4(708)$ & $11.4(116)$ & $2(2)$ & $40.8(416)$ & $56.8(577)$ & $2.7(27)$ & $1(1)$ & 83 & \\
\hline
\end{tabular}


Table 2. Cont.

\begin{tabular}{|c|c|c|c|c|c|c|c|c|c|c|c|}
\hline Variable & Groups & $\begin{array}{l}\text { Daily Fruit } \\
\text { Intake a \% } \\
(N)\end{array}$ & $\begin{array}{l}\text { No Daily Fruit } \\
\text { Intake }{ }^{b} \%(N)\end{array}$ & $\begin{array}{l}\text { No Fruit } \\
\text { Intake }{ }^{c} \% \\
(N)\end{array}$ & $\begin{array}{c}\text { Daily Fruit } \\
\text { Portions (Median } \\
\text { (IQR)) }\end{array}$ & $\begin{array}{c}\text { Daily } \\
\text { Vegetable } \\
\text { Intake }{ }^{\text {a } \%(N)}\end{array}$ & $\begin{array}{c}\text { No Daily } \\
\text { Vegetable } \\
\text { Intake } \text { b } \%(N)\end{array}$ & $\begin{array}{l}\text { No Vegetable } \\
\text { Intake }{ }^{c} \%(N)\end{array}$ & $\begin{array}{c}\text { Daily Vegetable } \\
\text { Portions (Median } \\
\text { (IQR)) }\end{array}$ & $\begin{array}{c}\text { Inadequate } \\
\text { FV Intake }{ }^{d} \\
(\%)\end{array}$ & $\begin{array}{c}\text { Chi } \\
\text { Squared } \\
\text { Test }\end{array}$ \\
\hline \multirow{2}{*}{$\begin{array}{l}\text { Alcohol } \\
\text { use }\end{array}$} & Not Daily & $15.3(1187)$ & $72.3(5615)$ & $12.4(962)$ & $2(2)$ & 43.9 (3409) & $54.6(4239)$ & $1.5(116)$ & $2(1)$ & 83 & \multirow[b]{2}{*}{$<0.001$} \\
\hline & Daily & $21.2(40)$ & $63.0(119)$ & $15.9(30)$ & $2(3)$ & $56.6(107)$ & $41.8(79)$ & $1.6(3)$ & $2(1)$ & 72 & \\
\hline \multirow{3}{*}{ Smoking } & Never & 15.7 (1119) & $72.5(5167)$ & $11.9(845)$ & $2(2)$ & $44.4(3164)$ & $54.3(3870)$ & $1.4(97)$ & $1(1)$ & 82 & \multirow{3}{*}{0.630} \\
\hline & Former & $12.2(30)$ & $71.8(176)$ & $15.9(39)$ & $2(2)$ & 46.9 (115) & $49.0(120)$ & $4.1(10)$ & $2(1)$ & 81 & \\
\hline & Current & $13.5(78)$ & $67.8(391)$ & $18.7(108)$ & $2(2)$ & $41.1(237)$ & $56.9(328)$ & $2.1(12)$ & $2(1)$ & 84 & \\
\hline \multirow{7}{*}{$\begin{array}{l}\text { Healthcare } \\
\text { use }\end{array}$} & No visits & $12.8(407)$ & $73.0(2322)$ & $14.2(451)$ & $2(2)$ & 38.0 (1208) & 60.4 (1919) & $1.7(53)$ & $2(1)$ & 85 & \multirow{7}{*}{$<0.001$} \\
\hline & One visit & $16(259)$ & 72.4 (1190) & $11.8(194)$ & $2(2)$ & $44.2(726)$ & $54.2(890)$ & $1.6(27)$ & $2(1)$ & 84 & \\
\hline & Two visits & $16.4(165)$ & 71.1 (714) & $12.5(125)$ & $2(2)$ & 47.7 (479) & 51.1 (513) & $1.2(12)$ & $1(1)$ & 82 & \\
\hline & Three visits & $16.7(156)$ & $73.2(684)$ & $10.2(95)$ & $2(2)$ & $48.3(452)$ & $50.7(474)$ & $1.0(9)$ & $2(1)$ & 83 & \\
\hline & Four visits & $18.0(106)$ & $73.2(432)$ & $8.8(52)$ & $2(2)$ & 50.7 (299) & $48.0(283)$ & $1.4(8)$ & $1(1)$ & 79 & \\
\hline & Five visits & $19.2(41)$ & $65.4(140)$ & $15.4(33)$ & 2 (3) & 53.7 (115) & $44.9(96)$ & $1.4(3)$ & $1(1)$ & 72 & \\
\hline & Six visits and more & $24.0(93)$ & $65.1(252)$ & $10.6(42)$ & $2(3)$ & $61.2(237)$ & $37.0(143)$ & $1.8(7)$ & $1(1)$ & 69 & \\
\hline
\end{tabular}

IQR: interquartile range. N/A: not applicable. The chi-squared test refers to the comparison of inadequate FV intake across categories of socio-demographic and lifestyle variables;

a Participants who reported daily consumption; ${ }^{b}$ Participants who reported consumption on a less than daily basis; ${ }^{\mathrm{c}}$ Participants who reported no consumption; ${ }^{\mathrm{d}}$ Participants who reported consumption of less than 5 portions of fruits and/or vegetables per day. 


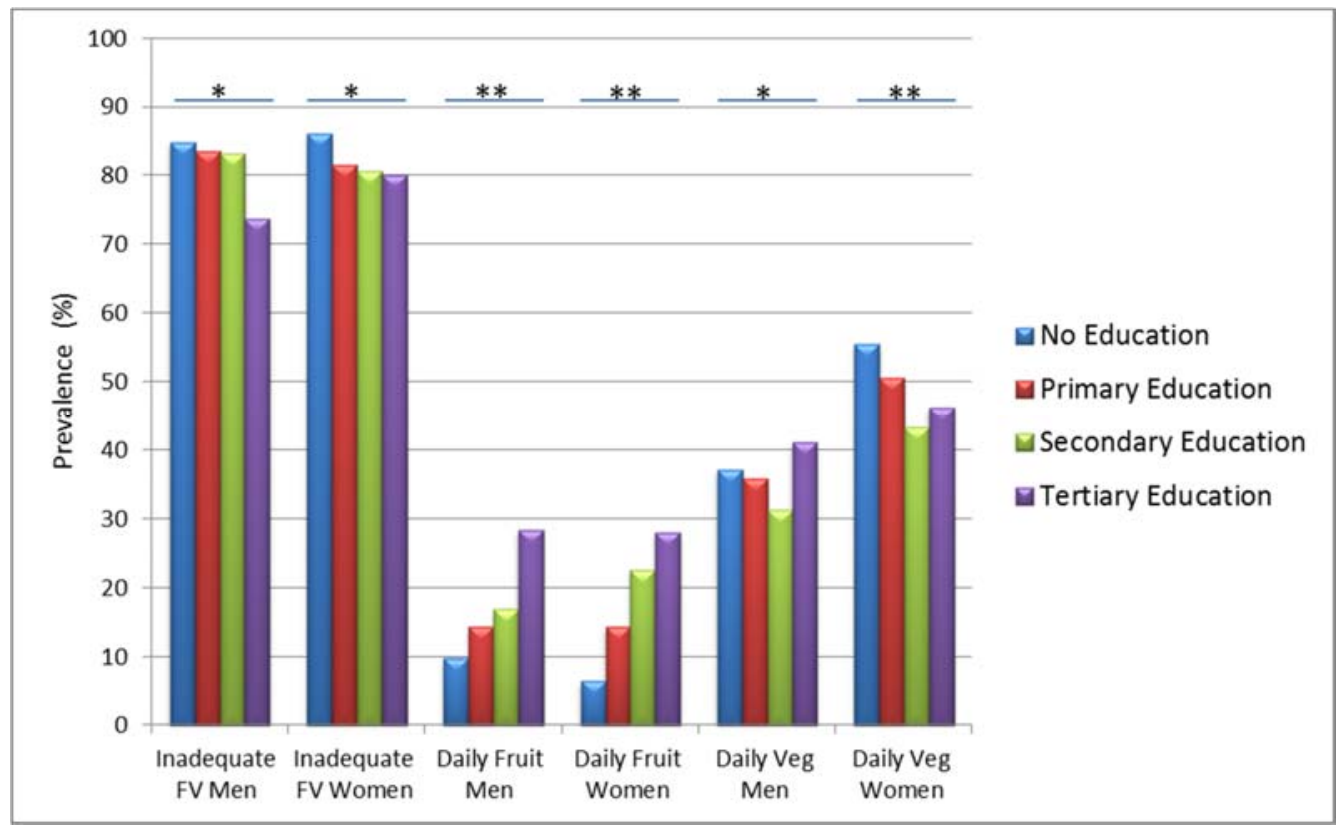

Figure 2. Prevalence of daily fruit, daily vegetable and inadequate fruits and vegetables (FV) intake among men and women in different education categories $(N=7953)$ * Significant differences in fruit or vegetable intake across different educational levels $(p<0.05) ;{ }^{* *}$ Significant differences in fruit or vegetable intake across different educational levels $(p<0.0001)$.

\subsection{Independent Association of FV Intake with Socio-demographic Characteristics}

Women were at a lesser risk for less than daily fruit (OR $=0.84,95 \% \mathrm{CI}: 0.73,0.96)$ and vegetable intake (OR $=0.51,95 \%$ CI: $0.46,0.56$ ) as well as inadequate FV (OR $=0.82,95 \%$ CI: $0.72,0.93)$ compared to men (Table 3). There was a strong association between the risk of less than daily vegetable intake and age. Older participants were less likely to have less than daily vegetable intake with OR of 0.54 ( $95 \%$ CI: $0.43,0.67)$ for the oldest age group compared to the youngest age group. Lower education was significantly associated with less than daily fruit intake but not vegetable intake. Odds of less than daily fruit intake decreased in those with primary education (OR $=0.55,95 \% \mathrm{CI}: 0.43,0.70)$ and those with secondary education ( $\mathrm{OR}=0.27,95 \% \mathrm{CI}: 0.18,0.40$ ) compared to those without any formal education. Employment and occupation were important in the overall risk of inadequate FV intake. Those with higher earning occupations like small (OR $=0.83,95 \%$ CI: $0.69,1.00)$, large business owners $(\mathrm{OR}=0.50,95 \% \mathrm{CI}: 0.32,0.83)$ and professionals (OR $=0.66,95 \% \mathrm{CI}: 0.50,0.86)$ were less likely to have inadequate FV intake compared to farmers. Migrants were at higher risk of less than daily fruit consumption as well as overall inadequate FV intake compared to non-migrants. Also, ethnic groups from Iringa (OR $=1.31,95 \%$ CI: 1.12, 1.52), Shinyanga/Mwanza/Tabora (OR $=1.78,95 \%$ CI: 1.45, 2.21), Kilimanjaro (OR $=1.39,95 \%$ CI: $1.06,1.82)$ and Mbeya (OR $=1.79,95 \%$ CI: $1.20,2.64)$ were more likely not to consume vegetables daily compared to those from Morogoro but there were no significant differences in their overall FV intake (Table 3). 
Table 3. Association of fruit and vegetable intake with socio-demographic characteristics $(N=7953)$.

\begin{tabular}{|c|c|c|c|c|c|c|c|}
\hline & & \multicolumn{2}{|c|}{$\begin{array}{l}\text { Risk for Less than Daily } \\
\text { Fruit Intake }\end{array}$} & \multicolumn{2}{|c|}{$\begin{array}{l}\text { Risk for Less than Daily } \\
\text { Vegetable Intake }\end{array}$} & \multicolumn{2}{|c|}{$\begin{array}{c}\text { Risk for Inadequate } \\
\text { Fruit and Vegetable } \\
\text { Intake }\end{array}$} \\
\hline & & OR * & $95 \% \mathrm{CI}$ & OR ** & $95 \% \mathrm{CI}$ & OR *** & $95 \% \mathrm{CI}$ \\
\hline \multirow{2}{*}{ Sex } & Men & Ref & - & Ref & - & Ref & - \\
\hline & Women & 0.84 & $0.73-0.96$ & 0.51 & $0.46-0.56$ & 0.82 & $0.72-0.93$ \\
\hline \multirow{2}{*}{ Age } & $<25$ years & Ref & - & Ref & - & Ref & - \\
\hline & $>60$ & 1.28 & $0.88-1.76$ & 0.54 & $0.43-0.67$ & 0.79 & $0.59-1.05$ \\
\hline \multirow{4}{*}{$\begin{array}{l}\text { Marital } \\
\text { Status }\end{array}$} & Never married/cohabiting & Ref & - & Ref & - & Ref & - \\
\hline & Monogamously married/cohabiting & 0.84 & $0.71-0.99$ & 0.88 & $0.77-0.99$ & 0.89 & $0.76-1.05$ \\
\hline & Polygamous married/cohabiting & 0.90 & $0.49-1.65$ & 0.75 & $0.48-1.15$ & 0.84 & $0.50-1.43$ \\
\hline & Widowed & 1.33 & $0.88-2.01$ & 0.86 & $0.67-1.11$ & 1.19 & $0.85-1.66$ \\
\hline $\begin{array}{l}\text { Education } \\
\text { Level }\end{array}$ & Tertiary & 0.27 & $0.19-0.40$ & 0.94 & $0.70-1.27$ & 0.61 & $0.43-0.88$ \\
\hline \multirow{7}{*}{ Occupation } & Farming/Livestock/Fishing & Ref & - & Ref & - & Ref & - \\
\hline & Small business & 0.69 & $0.56-0.84$ & 1.16 & $1.00-1.34$ & 0.84 & $0.69-1.00$ \\
\hline & Large business & 0.39 & $0.25-0.63$ & 0.75 & $0.49-1.15$ & 0.50 & $0.32-0.83$ \\
\hline & Professionals & 0.51 & $0.39-0.68$ & 1.00 & $0.79-1.28$ & 0.66 & $0.50-0.86$ \\
\hline & Skilled manual workers \& drivers & 0.61 & $0.48-0.78$ & 1.25 & $1.03-1.51$ & 0.87 & $0.68-1.09$ \\
\hline & Unskilled laborers \& bar workers & 0.90 & $0.65-1.25$ & 1.28 & $1.02-1.60$ & 1.26 & $0.92-1.72$ \\
\hline & Not working & 0.99 & $0.81-1.20$ & 1.46 & $1.27-1.66$ & 1.28 & $1.07-1.52$ \\
\hline \multirow{3}{*}{ Ethnicity } & Morogoro & Ref & - & Ref & - & Ref & - \\
\hline & Iringa & 1.03 & $0.83-1.28$ & 1.31 & $1.12-1.52$ & 1.08 & $0.88-1.32$ \\
\hline & Shinyanga/Mwanza/Tabora & 1.06 & $0.80-1.41$ & 1.78 & $1.45-2.21$ & 1.14 & $0.87-1.49$ \\
\hline \multirow{2}{*}{ Religion } & Lutheran & 0.69 & $0.46-1.03$ & 0.68 & $0.48-0.97$ & 1.12 & $0.70-1.77$ \\
\hline & Other \& No Religion & 0.99 & $0.75-1.29$ & 1.02 & $0.83-1.26$ & 0.98 & $0.75-1.27$ \\
\hline \multirow{2}{*}{$\begin{array}{l}\text { Migration } \\
\text { Status }\end{array}$} & Non-Migrant & Ref & - & Ref & - & Ref & - \\
\hline & Migrant & 1.15 & $1.00-1.31$ & 1.08 & $0.97-1.19$ & 1.14 & $1.00-1.30$ \\
\hline
\end{tabular}

All estimates were from a multivariable model adjusting for gender, age, marital status, educational level, occupation, ethnicity, religion and migration status. ${ }^{*} \mathrm{OR}>1$ and $\mathrm{OR}<1$, describes the increased and decreased likelihood to consume fruits less than daily respectively; ${ }^{* *} \mathrm{OR}>1$ and $\mathrm{OR}<1$, describes the increased and decreased likelihood to consume vegetables less than daily respectively; ${ }^{* * *} \mathrm{OR}>1$ and $\mathrm{OR}<1$, describes the increased and decreased likelihood to consume less than 5 portions fruits and vegetables daily respectively.

Participants who reported drinking alcohol on a daily basis were less at risk for less than daily fruit $(\mathrm{OR}=0.68, \mathrm{CI}: 0.47,0.98)$, vegetable $(\mathrm{OR}=0.68, \mathrm{CI}: 0.50,0.92)$ and inadequate FV consumption $(\mathrm{OR}=0.62$, CI: 0.44, 0.86) even after adjustment for all socio-demographic indicators and this was similar for men and women (Table 4). In contrast, no statistically-significant associations were observed between FV consumption and smoking.

We observed a strong association between inadequate FV intake and healthcare use in this cross-sectional study even after adjusting for various socio-demographic factors as well as smoking and alcohol consumption. . Higher healthcare service use seemed to be protective for inadequate FV intake. The results showed maximum protection for participants who reported $\geq 6$ visits where the odds of inadequate $\mathrm{FV}$ intake reduced by $59 \%$ compared to those without any healthcare visits (Figure 3). We did not observe any sex differences in the association between inadequate FV intake and healthcare use (result not shown). 
Table 4. Association of inadequate fruit and vegetable intake with smoking and alcohol consumption $(N=7953)$.

\begin{tabular}{|c|c|c|c|c|c|c|c|c|}
\hline & & & \multicolumn{2}{|c|}{$\begin{array}{l}\text { Risk for Less than } \\
\text { Daily Fruit Intake }\end{array}$} & \multicolumn{2}{|c|}{$\begin{array}{l}\text { Risk for Less than Daily } \\
\text { Vegetable Intake }\end{array}$} & \multicolumn{2}{|c|}{$\begin{array}{l}\text { Risk for Inadequate Fruit } \\
\text { and Vegetable Intake }\end{array}$} \\
\hline & & & OR * & $95 \%$ CI & OR ** & $95 \%$ CI & OR $* * *$ & $95 \%$ CI \\
\hline \multirow{5}{*}{$\begin{array}{c}\text { All } \\
\text { subjects }\end{array}$} & \multirow{3}{*}{ Smoking status } & Never & Ref & - & Ref & - & Ref & - \\
\hline & & Former & 1.1 & $0.70-1.61$ & 0.98 & $0.74-1.29$ & 0.91 & $0.64-1.28$ \\
\hline & & Current & 1.00 & $0.76-1.31$ & 0.68 & $0.50-0.92$ & 1.05 & $0.82-1.35$ \\
\hline & \multirow{2}{*}{$\begin{array}{l}\text { Alcohol } \\
\text { consumption }\end{array}$} & Not daily & Ref & - & Ref & - & Ref & - \\
\hline & & Daily & 0.68 & $0.47-0.98$ & 0.68 & $0.50-0.92$ & 0.62 & $0.44-0.86$ \\
\hline \multirow{5}{*}{ Men } & \multirow{3}{*}{ Smoking status } & Never & Ref & - & Ref & - & Ref & - \\
\hline & & Former & 0.96 & $0.62-1.51$ & 0.85 & $0.61-1.18$ & 0.84 & $0.57-1.26$ \\
\hline & & Current & 1.01 & $0.75-1.36$ & 0.94 & $0.75-1.17$ & 0.97 & $0.74-1.20$ \\
\hline & \multirow{2}{*}{$\begin{array}{c}\text { Alcohol } \\
\text { consumption }\end{array}$} & Not daily & Ref & - & Ref & - & Ref & - \\
\hline & & Daily & 0.85 & $0.45-1.47$ & 0.59 & $0.38-0.90$ & 0.83 & $0.50-1.39$ \\
\hline \multirow{5}{*}{ Women } & \multirow{3}{*}{ Smoking status } & Never & Ref & - & Ref & - & Ref & - \\
\hline & & Former & 3.36 & $0.79-14.32$ & 1.39 & $0.82-2.35$ & 1.19 & $0.57-2.49$ \\
\hline & & Current & 1.52 & $0.64-3.59$ & 1.17 & $0.75-1.82$ & 1.83 & $0.90-3.73$ \\
\hline & \multirow{2}{*}{$\begin{array}{c}\text { Alcohol } \\
\text { consumption }\end{array}$} & Not daily & Ref & - & Ref & - & Ref & - \\
\hline & & Daily & 0.57 & $0.35-0.95$ & 0.79 & $0.52-1.20$ & 0.48 & $0.31-0.74$ \\
\hline
\end{tabular}

All estimates were from a mutually-adjusted model, additionally adjusted for socio-demographic characteristics (gender, age, marriage, education, occupation, ethnicity, religion and migration). ${ }^{*} \mathrm{OR}>1$ and $\mathrm{OR}<1$, describes the increased and decreased likelihood to consume fruits less than daily respectively; ${ }^{* *} \mathrm{OR}>1$ and $\mathrm{OR}<1$, describes the increased and decreased likelihood to consume vegetables less than daily respectively; ${ }^{* * *}$ OR $>1$ and $\mathrm{OR}<1$, describes the increased and decreased likelihood to consume less than 5 portions fruits and vegetables daily respectively.

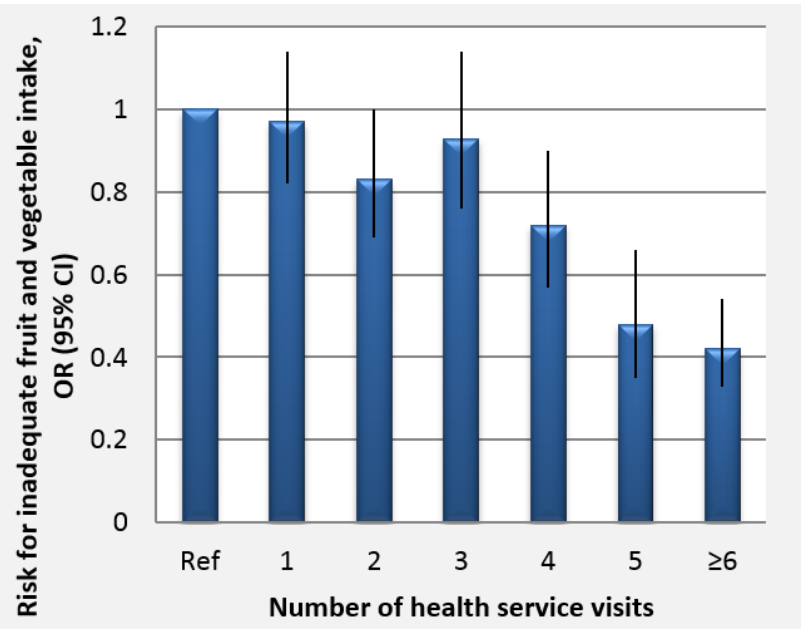

Figure 3. Odds ratios and confidence intervals for the association of inadequate fruit and vegetable intake with frequency of healthcare use in the previous 12 months (cumulate number of outpatient, health dispensary and home visits) $N=7953$.

\section{Discussion}

In line with findings from other STEPs surveys done in the sub-Saharan African region [15-19], most of these rural and semi-urban Tanzanian study participants did not meet the five portions daily FV consumption recommendation. More effort is needed to increase the frequency of fruit intake than is needed to increase the frequency of vegetable intake. This is because almost half of the participants consumed vegetables daily while a lesser fraction consumed fruits daily. An important target group for nutritional recommendation is persons of lower SES.

We generally observed that vegetables are widely consumed but in very small portions while fruits are mainly consumed in larger portions, especially among those with higher SES. Daily vegetable consumption was three-fold more prevalent when compared to daily fruit intake. Furthermore, those 
who had not eaten a fruit at all in the past 7 days of the interview were almost ten times more $(12.7 \%)$ than those who had not eaten vegetables $(1.5 \%)$ at all. Our finding of a higher consumption of vegetables than fruits is supported by evidence from studies in South Africa and Mozambique [18,20]. Vegetables are a cheap relish that accompanies daily staples like stiff polenta ("Ugali"), rice, flat bread and others [21,50] and this may explain to a large extent, the high daily vegetable consumption.

Although inadequate FV intake was not different between men and women, it was evident in our findings that men were $49 \%$ less likely to consume vegetables daily. This could be due to gender differences in health consciousness or gender roles which may give women more access to food supplies [51-53] as well as the fact that men tend to consume more out of home prepared meals especially for lunch while at work [54], most of which is low on vegetables.

Epidemiologic transition in the semi-urban settings where particularly younger people are exposed to more western-style diets may explain the observed decline of daily vegetable intake as well as portions sizes with decreasing age. Although participants under 18 years are more likely to be financially dependent, thus depending on parental food choices, the observed protective age effect was also made among subjects older than 25 years. This age group ( $<18$ years) comprised only $10 \%$ of our study sample, thus, we expect minimal bias of our results due to their inclusion. In rural settings, older people have shown more attachment to vegetables as their source of nutrition, being that they are mostly farmers with a wealth of knowledge on vegetable varieties and use $[50,55]$. Interestingly, in this study we did not observe farmers to have high vegetable intake.

For fruit intake, cost is a clear barrier in many poor settings similar to Tanzania [56,57]. Our findings demonstrate the importance of SES to fruit intake. Higher level of education and high-income occupations were facilitators of more frequent and larger portions of fruit consumption. It has been previously reported that affordability of fruits is an important bottleneck for their consumption $[18,20,54]$. Unlike vegetables, the relationship between income and affordability of fruits has received global attention $[13,56]$. The relative cost of fruits in low income countries has been reported to be 50 times higher than the relative costs in high income countries [58]. Fruits are easy-to-perish commodities and often lack controlled environments for quality storage, transportation as well as packing. This leads to large post-harvest losses and results in high market prices of fruits [59].

Cultural perceptions on FV intake differ. Contrary to our findings on vegetable intake, the oldest participants consumed least fruits. This may be explained by attitudes towards fruits as demonstrated by another study in neighboring Zanzibar where fruits are regarded as snacks for children [22,60]. Another study conducted in rural Tanzania, also reported that children consumed fruits the most [61]. The difference in association between fruit intake and age groups across countries in different continents [62-64] suggests culture to be an important context that needs to be considered in developing prevention programs.

This study also found that frequent health care use was associated with more FV consumption. Even though we do not have information on reasons for health care use, it is more likely that those with more health care use are more health conscious and may therefore benefit from preventive health advice including FV consumption. Exploratory analyses suggested that participants with higher SES were more likely to have higher healthcare use thus, health education or promotions aimed at people most vulnerable to inadequate FV intake, should rely more on community-based approaches rather than health facilities as the delivery channel. This, in addition, provides an opportunity for public health interventions to address both inadequate FV intake and poor health service use through integrated approaches. Our observation of higher consumption of FV among those who drank more alcohol is supported by previous studies which showed that individuals tend to compensate an unhealthy lifestyle with another healthy one [41]. Interestingly, this was not true for smoking in our study.

\section{Strengths and Limitations}

The strengths of this study derive from being the first study to the best of our knowledge from Tanzania providing detailed description about patterns of FV intake and socio-demographic and 
lifestyle determinants. This study also contributes in detail, to the growing literature on the burden of NCD risk factors in Africa. Our sample size is large and representative of the IU-HDSS area. The MZIMA cohort includes 70\% of eligible adults from the HDSS area [46] whereas our sample includes 92\% of the MZIMA cohort. All ethnic groups, religious affiliations and other socio-demographic attributes of the area were well-captured in our sample. We explored FV intake both separately and in combination according to WHO recommendations. The diverse cultural make-up makes these findings generalizable to other small towns with similar settings. Being nested within the IU-HDSS, a part of the INDEPTH network and as the NCD research agenda is building up in the African continent, our findings will make a useful source of comparison to similar studies in the future in Tanzania and beyond. Our study also has limitations. First, it was a cross-sectional study and precludes drawing conclusions about causal associations. Second, there may have been recall bias in the responses for FV intake. However, the recall period was short (seven days) hence, we expect minimal recall bias. The short recall period in this setting is also likely representative of longer-term dietary habits. Second, there was lack of information on some of the possible confounders or modifiers, including awareness about importance of FV intake, actual purchasing power and availability. These findings represent data collected over one year and no specific seasonal analysis was done.

\section{Conclusions}

FV consumption in the study setting was associated with SES and cultural patterns. Most immediate gain in improving overall FV consumption in resource-poor settings may be attained by promoting daily consumption of vegetables and increase in number of standard portions of vegetables consumed. Improving access to fruits by making them more affordable may contribute to improving intake rates. More qualitative and quantitative research are needed to better understand the prevalent knowledge, attitude and perception of fruits and vegetable consumption in local cultural contexts in order to improve their intake rates in these settings. Given the importance of awareness in health behavior change, more effort is also needed in the dissemination of the message regarding the number of FV portions necessary to maintain good health.

Acknowledgments: We are thankful to Swiss Tropical and Public Health Institute (Swiss TPH), whose financial and technical support helped in the realization of this manuscript. We are also grateful to Netherlands Initiative for Capacity Building in Higher Education (NICHE) as well as Global Fund for AIDS, TB and Malaria who funded the design and implementation of the MZIMA Cohort in Ifakara. We thank all Ifakara Health Institute (IHI) MZIMA staff and study participants of MZIMA round 1. We also wish to thank Christian Schindler of Swiss TPH, for his valuable support with the statistical analyses.

Author Contributions: B.M. conceived and designed the study, analyzed the data and wrote the manuscript. I.C.E. contributed towards interpretation of the data and writing of the manuscript. R.A. oversaw data collection and did the initial data handling including data set cleaning and generation of needed categories. S.A., P.K., M.T., R.K. critically reviewed the manuscript. E.G. contributed towards conceiving and designing the study and also reviewed the manuscript. N.P.-H. contributed to data analysis, interpretation and writing of the manuscript. All authors read and approved the final version of the manuscript.

Conflicts of Interest: The authors declare no conflict of interest. The funders/sponsors had no role in the design of the study; in the collection, analyses, or interpretation of data; in the writing of the manuscript and in the decision to publish the results.

\section{References}

1. Nishida, C.; Uauy, R.; Kumanyika, S.; Shetty, P. The Joint WHO/FAO Expert Consultation on diet, nutrition and the prevention of chronic diseases: Process, product and policy implications. Public Health Nutr. 2007, 7, 245-250. [CrossRef]

2. Boeing, H.; Bechthold, A.; Bub, A.; Ellinger, S.; Haller, D.; Kroke, A.; Leschik-Bonnet, E.; Muller, M.J.; Oberritter, H.; Schulze, M.; et al. Critical review: Vegetables and fruit in the prevention of chronic diseases. Eur. J. Nutr. 2012, 51, 637-663. [CrossRef] [PubMed]

3. Slavin, J.L.; Lloyd, B. Health benefits of fruits and vegetables. Adv. Nutr. 2012, 3, 506-516. [CrossRef] [PubMed] 
4. Liu, R.H. Health-promoting components of fruits and vegetables in the diet. Adv. Nutr. 2013, 4, 384S-392S. [CrossRef] [PubMed]

5. Esfahani, A.; Wong, J.M.W.; Truan, J.; Villa, C.R.; Mirrahimi, A.; Srichaikul, K.; Kendall, C.W.C. Health Effects of Mixed Fruit and Vegetable Concentrates: A Systematic Review of the Clinical Interventions. J. Am. Coll. Nutr. 2011, 30, 285-294. [CrossRef] [PubMed]

6. Halliwell, B. Oxidative stress and cancer: Have we moved forward? Biochem. J. 2007, 401, 1-11. [CrossRef] [PubMed]

7. Homocysteine Lowering Trialists' Collaboration. Dose-dependent effects of folic acid on blood concentrations ofhomocysteine: A meta-analysis of the randomized trials. Am. J. Clin. Nutr. 2005, 82, 806-812.

8. Malinow, M.R.; Bostom, A.G.; Krauss, R.M. Homocyst(e)ine, Diet, and Cardiovascular Diseases: A statement for healthcare professionals from the Nutrition Committee, American Heart Association. Circulation 1999, 99, 178-182. [CrossRef] [PubMed]

9. Filippini, T.; Violi, F.; D'Amico, R.; Vinceti, M. The effect of potassium supplementation on blood pressure in hypertensive subjects: A systematic review and meta-analysis. Int. J. Cardiol. 2017, 230, 127-135. [CrossRef] [PubMed]

10. Blanch, N.; Clifton, P.M.; Keogh, J.B. A systematic review of vascular and endothelial function: Effects of fruit, vegetable and potassium intake. Nutr. Metab. Cardiovasc. Dis. 2015, 25, 253-266. [CrossRef] [PubMed]

11. Wang, X.; Ouyang, Y.; Liu, J.; Zhu, M.; Zhao, G.; Bao, W.; Hu, F.B. Fruit and vegetable consumption and mortality from all causes, cardiovascular disease, and cancer: Systematic review and dose-response meta-analysis of prospective cohort studies. Br. Med. J. 2014, 349, g4490. [CrossRef] [PubMed]

12. He, F.J.; Nowson, C.A.; Lucas, M.; MacGregor, G.A. Increased consumption of fruit and vegetables is related to a reduced risk of coronary heart disease: Meta-analysis of cohort studies. J. Hum. Hypertens. 2007, 21, 717-728. [CrossRef] [PubMed]

13. Hall, J.N.; Moore, S.; Harper, S.B.; Lynch, J.W. Global variability in fruit and vegetable consumption. Am. J. Prev. Med. 2009, 36, 402-409. [CrossRef] [PubMed]

14. World Health Organization (WHO). Global Health Risks: Mortality and Burden of Disease Attributable to Selected Major Risks; WHO: Geneva, Switzerland, 2009; pp. 1-70.

15. Mayige, M.; Kagaruki, G. Tanzania STEPS Survey Report; National Institute for Medical Research: Dar Es Salaam, Tanzania, 2013; pp. 1-154.

16. Ngwira, B.; Kandoole, N.; Mvula, C.; Muwalo, B.; Msyamboza, K.; Chitimbe, G.; Chikhata, M.; Kubwalo, H.; Mwase, Y.; Saukila, K.; et al. Malawi National STEPS Survey for Chronic Non Communicable Diseases and Their Risk Factors; Ministry of Health: Malawi, Africa, 2010; pp. 1-131.

17. Ministry of Health (MoH); World Health Organization (WHO). Prevalence Rates of the Common Non-Communicable Diseases and Their Risk Factors in Lusaka District Zambia 2008; Ministry of Health and WHO: Lusaka, Zambia, 2008; pp. 1-72.

18. Padrao, P.; Laszczynska, O.; Silva-Matos, C.; Damasceno, A.; Lunet, N. Low fruit and vegetable consumption in Mozambique: Results from a WHO STEPwise approach to chronic disease risk factor surveillance. $\mathrm{Br}$. J. Nutr. 2012, 107, 428-435. [CrossRef] [PubMed]

19. Ministry of Health (MoH). Republic of Botswana Chronic Disease Risk Factors Surveillance Report 2007; Ministry of Health: Gaborone, Botswana, 2007; pp. 1-142.

20. Peltzer, K.; Phaswana-Mafuya, N. Fruit and vegetable intake and associated factors in older adults in South Africa. Glob. Health Action 2012, 5, 1-8. [CrossRef] [PubMed]

21. Ruel, M.; Minot, N.; Smith, L. Patterns and Determinants of Fruit and Vegetable Consumption in Sub-Saharan Africa; World Health Organization: Geneva, Switzerland, 2005; pp. 1-45.

22. Keller, A. Association between Fruit and Vegetables Eating Habits and Practices in Relation to the Prevalence of Diet Related Chronic Non Communicable Diseases in Zanzibar Tanzania; University of Copenhagen: Copenhagen, Denmark, 2012.

23. Glasson, C.; Chapman, K.; James, E. Fruit and vegetables should be targeted separately in health promotion programmes: Differences in consumption levels, barriers, knowledge and stages of readiness for change. Public Health Nutr. 2011, 14, 694-701. [CrossRef] [PubMed]

24. Guillaumie, L.; Godin, G.; Vezina-Im, L.A. Psychosocial determinants of fruit and vegetable intake in adult population: A systematic review. Int. J. Behav. Nutr. Phys. Act. 2010, 7, 12. [CrossRef] [PubMed] 
25. Love, P.; Maunder, E.; Green, M.; Ross, F.; Smale-Lovely, J. South African Food-Based Dietary Guidelines: Testing of the Preliminary Guidelines among Women in Kwa Zulu Natal and Western Cape. South Afr. J. Clin. Nutr. 2001, 14, 9-19.

26. Naude, C. "Eat plenty of vegetables and fruit every day": A food-based dietary guideline for South Africa. South Afr. J. Clin. Nutr. 2013, 26, S46-S56.

27. Ministry of Health (MoH-Kenya). National Guidelines for Healthy Diets and Physical Activity; Ministry of Health: Nairobi, Kenya, 2017.

28. Ministry of Health (MoH-Malawi). National Nutrition Guidelines for Malawi; Ministry of Health: Lilongwe, Malawi, 2007; pp. 1-49.

29. Ministry of Health (MoH-Nigeria). Food-Based Dietary Guidelines for Nigeria-A Guide to Healthy Eating; Federal Ministry of Health: Abuja, Nigeria, 2006; pp. 1-36.

30. Bandura, A. Social Cognitive Theory of Self Regulation. Organ. Behav. Hum. Decis. Process. 1991, 50, $248-287$. [CrossRef]

31. Erinosho, T.O.; Moser, R.P.; Oh, A.Y.; Nebeling, L.C.; Yaroch, A.L. Awareness of the Fruits and Veggies-More Matters campaign, knowledge of the fruit and vegetable recommendation, and fruit and vegetable intake of adults in the 2007 Food Attitudes and Behaviors (FAB) Survey. Appetite 2012, 59, 155-160. [CrossRef] [PubMed]

32. Metta, E.; Msambichaka, B.; Mwangome, M.; Nyato, D.J.; Dieleman, M.; Haisma, H.; Klatser, P.; Geubbels, E. Public policy, health system, and community actions against illness as platforms for response to NCDs in Tanzania: A narrative review. Glob. Health Action 2014, 7, 23439. [CrossRef] [PubMed]

33. Kamphuis, C.B.; Giskes, K.; de Bruijn, G.J.; Wendel-Vos, W.; Brug, J.; van Lenthe, F.J. Environmental determinants of fruit and vegetable consumption among adults: A systematic review. Br. J. Nutr. 2006, 96, 620-635. [PubMed]

34. Peltzer, K.; Pengpid, S. Fruits and vegetables consumption and associated factors among in-school adolescents in seven African countries. Int. J. Public Health 2010, 55, 669-678. [CrossRef] [PubMed]

35. Hosseinpoor, A.; Bergen, N.; Kunst, A.; Harper, S.; Guthold, R.; Rekve, D.; d'Espaignet, E.; Naidoo, N.; Chatterji, S. Socioeconomic inequalities in risk factors for noncommunicable diseases in low-income andmiddle-income countries: Results from the WorldHealth Survey. BMC Public Health 2012, 12, 912.

36. Mikkonen, J.; Raphael, D. Social Determinants of Health: The Canadian Facts; York University School of Health Policy and Management: Toronto, ON, Canada, 2010.

37. Wolf, R.L.; Lepore, S.J.; Vandergrift, J.L.; Wetmore-Arkader, L.; McGinty, E.; Pietrzak, G.; Yaroch, A.L. Knowledge, barriers, and stage of change as correlates of fruit and vegetable consumption among urban and mostly immigrant black men. J. Am. Diet. Assoc. 2008, 108, 1315-1322. [CrossRef] [PubMed]

38. Peltzer, K.; Promtussananon, S. Knowledge, barriers, and benefits of fruit and vegetable consumption and lay conceptions of nutrition among rural and semi-urban Black South Africans. Psychol. Rep. 2004, 94, 976-982. [CrossRef] [PubMed]

39. Shaikh, A.R.; Yaroch, A.L.; Nebeling, L.; Yeh, M.C.; Resnicow, K. Psychosocial predictors of fruit and vegetable consumption in adults a review of the literature. Am. J. Prev. Med. 2008, 34, 535-543. [CrossRef] [PubMed]

40. Salehi, L.; Eftekhar, H.; Mohammad, K.; Tavafian, S.S.; Jazayery, A.; Montazeri, A. Consumption of fruit and vegetables among elderly people: A cross sectional study from Iran. Nutr. J. 2010, 9, 2. [CrossRef] [PubMed]

41. Giles, E.L.; Brennan, M. Trading between healthy food, alcohol and physical activity behaviours. BMC Public Health 2014, 14, 1231. [CrossRef] [PubMed]

42. Lahti-Koski, M.; Pietinen, P.; Heliovaara, M.; Vartiainen, E. Associations of body mass index and obesity with physical activity, food choices, alcohol intake, and smoking in the 1982-1997 FINRISK Studies. Am. J. Clin. Nutr. 2002, 75, 809-817. [CrossRef] [PubMed]

43. Johnson, M.F.; Nichols, J.F.; Sallis, J.F.; Calfas, K.J.; Hovell, M.F. Interrelationships between physical activity and other health behaviors among university women and men. Prev. Med. 1998, 27, 536-544. [CrossRef] [PubMed]

44. Lee, I.C.; Chang, C.S.; Du, P.L. Do healthier lifestyles lead to less utilization of healthcare resources? BMC Health Serv. Res. 2017, 17, 243. [CrossRef] [PubMed] 
45. Geubbels, E.; Amri, S.; Levira, F.; Schellenberg, J.; Masanja, H.; Nathan, R. Health \& Demographic Surveillance System Profile: The Ifakara Rural and Urban Health and Demographic Surveillance System (Ifakara HDSS). Int. J. Epidemiol. 2015, 44, 848-861. [PubMed]

46. Abdul, R.; Ramaiya, A.; Mtenga, S.; Mtowa, A.; Geubbels, E. MZIMA Longitudinal Cohort Study Progress Report: Serosurvey Round 1 June 2012-May 2013; IHI: Dar Es Salaam, Tanzania, 2014; pp. 1-38.

47. Boriello, G.; Schilit, B.; Lerer, A.; Chin, J. Open Data Kit. Retrieved 2017, from Open Data Kit Downloads. Available online: https:/ / opendatakit.org (accessed on 11 August 2017).

48. World Health Organization (WHO). The WHO STEPS Surveillance Manual; WHO: Geneva, Switzerland, 2005.

49. LSHTM Alpha Network: Analysing Longitudinal Population-Based HIV/AIDS Data in Africa. Available online: http:/ / www.alpha.lshtm.ac.uk (accessed on 11 August 2017).

50. Smith, F.; Eyzaguirre, P. African Leafy Vegetables: Their Role in the World Health Organization's Global Fruit and Vegetables Initiative. Afr. J. Food Agric. Nutr. Dev. 2007, 7, 1-17.

51. Girard, A.W.; Self, J.L.; McAuliffe, C.; Olude, O. The effects of household food production strategies on the health and nutrition outcomes of women and young children: A systematic review. Paediatr. Perinat. Epidemiol. 2012, 26, 205-222. [CrossRef] [PubMed]

52. Beardsworth, A.; Bryman, A.; Keil, T.; Goode, J.; Haslam, C.; Lancashire, E. Women, men and food: The significance of gender for nutritional attitudes and choices. Br. Food J. 2002, 104, 470-491. [CrossRef]

53. Quisumbing, A.; Brown, L.; Feldstein, H.; Haddad, L.; Pena, C.M. Women: The Key to Food Security; The International Food Policy Research institute: Washington, DC, USA, 1995; pp. 1-26.

54. Krige, S.M.; Mahomoodally, F.M.; Subratty, A.H.; Ramasawmy, D. Relationship between Socio-Demographic Factors and Eating Practices in a Multicultural Society. Food Nutr. Sci. 2012, 3, 286-295. [CrossRef]

55. Owouor, O.; Olaimer-Anyara, E. The value of leafy vegetables: An exploration of African Folkore. Afr. J. Food Agric. Nutr. Dev. 2007, 7, 1-13.

56. Miller, V.; Yusuf, S.; Chow, C.K.; Dehghan, M.; Corsi, D.J.; Lock, K.; Popkin, B.; Rangarajan, S.; Khatib, R.; Lear, S.A.; et al. Availability, affordability, and consumption of fruits and vegetables in 18 countries across income levels: Findings from the Prospective Urban Rural Epidemiology (PURE) study. Lancet Glob. Health 2016, 4, e695-e703. [CrossRef]

57. Mayen, A.L.; Marques-Vidal, P.; Paccaud, F.; Bovet, P.; Stringhini, S. Socioeconomic determinants of dietary patterns in low- and middle-income countries: A systematic review. Am. J. Clin. Nutr. 2014, 100, 1520-1531. [CrossRef] [PubMed]

58. Van Bakel, M.M.; Slimani, N.; Feskens, E.J.; Du, H.; Beulens, J.W.; van der Schouw, Y.T.; Brighenti, F.; Halkjaer, J.; Cust, A.E.; Ferrari, P.; et al. Methodological challenges in the application of the glycemic index in epidemiological studies using data from the European Prospective Investigation into Cancer and Nutrition. J. Nutr. 2009, 139, 568-575. [CrossRef] [PubMed]

59. USAID-TRADE-HUB. The Fresh Fruit and Vegetable Markets of East Africa: An Assessment of Regional Value Chain Actors Activities and Constraints in Kenya, Tanzania, Uganda; USAID: East Africa, 2013; pp. 1-87.

60. Keller, A.; de Courten, M.; Dræbel, T.A. Fruit and vegetable consumption and prevalence of diet-related chronic non-communicable diseases in Zanzibar, Tanzania: A mixed methods study. Lancet 2012, 380, S16. [CrossRef]

61. Msuya, T.S.; Kideghesho, J.R.; Luoga, E. Consumption of Indeginous Fruits in Uluguru North and Ruvu North Forest Reserves, Tanzania. Tanzan. J. For. Nat. Conserv. 2004, 75, 65-73.

62. Chong, K.H.; Lee, S.T.; Ng, S.A.; Khouw, I.; Poh, B.K. Fruit and Vegetable Intake Patterns and Their Associations with Sociodemographic Characteristics, Anthropometric Status and Nutrient Intake Profiles among Malaysian Children Aged 1-6 Years. Nutrients 2017, 9, 723. [CrossRef] [PubMed]

63. Rasmussen, M.; Krolner, R.; Klepp, K.I.; Lytle, L.; Brug, J.; Bere, E.; Due, P. Determinants of fruit and vegetable consumption among children and adolescents: A review of the literature. Part I: Quantitative studies. Int. J. Behav. Nutr. Phys. Act. 2006, 3, 22. [CrossRef] [PubMed]

64. Lorson, B.A.; Melgar-Quinonez, H.R.; Taylor, C.A. Correlates of fruit and vegetable intakes in US children. J. Am. Diet. Assoc. 2009, 109, 474-478. [CrossRef] [PubMed]

(C) 2018 by the authors. Licensee MDPI, Basel, Switzerland. This article is an open access article distributed under the terms and conditions of the Creative Commons Attribution (CC BY) license (http:/ / creativecommons.org/licenses/by/4.0/). 